\title{
Losartan Treatment Could Improve the Outcome of TBI Mice
}

\author{
Jianhua Xiong *t, Yalong Gao ${ }^{+}$, Xiaotian $\mathrm{Li}^{\dagger}$, Kai Li, Qifeng Li, Jun Shen, Zhenying Han and \\ Jianning Zhang*
}

Department of Neurosurgery, Tianjin Medical University General Hospital, Tianjin, China

Traumatic brain injury frequently leads to serious mortality and physical disability, yet effective treatments remains insufficient. TBI always leads to a series of secondary brain injuries including neuronal apoptosis, continuous inflammation, endoplasmic reticulum stress, and disruption of the blood-brain barrier. Sartans that block angiotensin II type 1 receptors are strongly neuroprotective, neurorestorative and anti-inflammatory. However, whether losartan, a FDA-approved and widely used drug for regulating blood pressure, is

OPEN ACCESS

Edited by:

Jia Lu,

DSO National Laboratories, Singapore

Reviewed by:

Andrew K. Ottens,

Virginia Commonwealth University,

United States

Sonia Villapol,

Houston Methodist Research Institute,

United States

*Correspondence:

Jianhua Xiong

doctorxiong@hotmail.com

Jianning Zhang

profzhang@hotmail.com

tThese authors have contributed equally to this work

Specialty section:

This article was submitted to Neurotrauma,

a section of the journal

Frontiers in Neurology

Received: 23 February 2020

Accepted: 29 July 2020

Published: 15 October 2020

Citation:

Xiong J, Gao Y, Li X, Li K, Li Q,

Shen J, Han Z and Zhang J (2020) Losartan Treatment Could Improve the

Outcome of TBI Mice.

Front. Neurol. 11:992.

doi: 10.3389/fneur.2020.00992 beneficial for improving the prognosis of TBI need more evidence. Through a controlled cortical impact injury mice model, we confirmed that losartan treatment could ameliorate $\mathrm{CCl}$-induced secondary brain injury. We found that losartan treatment decreased brain lesion volume, neuronal apoptosis and ER stress protein ATF4 and elF2 $\alpha$. Moreover, our results showed that losartan also improved neurological and motor function. It is worth pointing out that losartan increased the expression of tight junction proteins ZO-1 and alleviated brain edema and blood brain barrier leakage. Additionally, losartan inhibited pro-inflammatory factor TNF- $\alpha$ and improve anti-inflammatory factor IL-10. Taken together, our data demonstrated that losartan could improve the prognosis of $\mathrm{TBI}$ and may be a promising therapeutic method for mitigating TBI.

Keywords: traumatic brain injury, blood brain barrier, endoplasmic reticulum stress, neuroinflammation, angiotensin II type 1 receptors blockage, tight junction proteins, losartan

\section{INTRODUCTION}

Because of its high mortality and disability, traumatic brain injury (TBI) is a serious worldwide public health and socioeconomic problem $(1,2)$. Although there are limited high-quality epidemiological data, $\sim 5.3$ million people in the USA suffer from a TBI-related disability (3). However, there are currently no effective drugs for treatment of TBI. The pathogenesis of TBI is complicated and includes primary and secondary injury. Primary injury is characterized by immediate mechanical stress and loss of brain tissue after trauma (4). Secondary injury involves intricate cellular and biochemical pathological events, including oxidative stress, neuroinflammation, blood-brain barrier (BBB) damage, endoplasmic reticulum (ER) stress, brain edema, and neuronal apoptosis, which can occur within minutes after TBI and can last for hours to days to months (5-8).

Excessive Angiotensin II (AngII) increases reactive oxygen species through AngII type 1 receptor (AT1R)-mediated signaling in various disorders, including hypertension, stroke, and coronary heart disease (9-12). Overactivation of the AT1R was reported to be closely related to secondary damage after TBI (13-15). Moreover, AngII-mediated oxidative signaling can be blocked by AT1R antagonists $(16,17)$. For example, Villapol et al. reported that candesartan and 
telmisartan, which can both block AT1R and activate peroxisome proliferator-activated receptor- $\gamma$ (PPAR $\gamma$ ), have therapeutic potential in TBI (18). In recent years, studies have found that the neuroprotective effects of AngII receptor blocker (ARB) drugs include modulation of microglial activation states (19), alleviating endoplasmic reticulum (20), inhibition of neuronal apoptosis (21) and inhibition of overproduction of inflammatory factors (22) and so on. Related mechanisms such as downregulation of the TLR2 signaling pathway (23), PPAR $\gamma$ activation (18), activating Wnt/ $\beta$-catenin signaling (24) and so on. Comparison of the protective effects of different sartans on TBI mice can be found in Table 1 .

Furthermore, there is increasing evidence that AT1R antagonists are neuroprotective and anti-inflammatory (27). Nevertheless, few studies have examined the use of losartan as a therapeutic agent for reducing neuroinflammation, edema, and lesion volume following TBI.

Losartan is an FDA-approved drug used to treat hypertension (28), and acts as an AT1R antagonist to selectively block AngII from binding to the AT1R (29). Losartan was previously reported to block brain TGF- $\beta$ signaling and prevent epilepsy in albumin and $\mathrm{BBB}$ breakdown models of epileptogenesis (26). Losartan was also neuroprotective following TBI in mice (25), and reduced hippocampal CA1 injury following cerebral ischemia/reperfusion in rats (30). Furthermore, AT1R blockade had direct neuroprotective actions in cultured microglia $(16,31)$. Mechanistically, the neuroprotective actions of sartans following TBI in mice were reported to involve activation of the PPAR $\gamma$ pathway (18). However, the therapeutic effects and mechanisms of action of losartan remain to be validated. Thus, the aim of the present study was to examine the efficacy of losartan in alleviating the pathological and neurobehavioral deficits observed following TBI in mice.

\section{MATERIALS AND METHODS}

\section{Experimental Groups and Drug Administration}

Adult male C57BL/6 mice (8-10 weeks old, 22-25g) were purchased from the Experimental Animal Laboratories of the Academy of Military Medical Sciences (Beijing, China). The dose of losartan (losartan potassium tablet, dissolved in $0.9 \%$ saline) was based on that previously reported $(32,33)$. Mice were randomly assigned to receive losartan $(3 \mathrm{mg} / \mathrm{kg})$ or vehicle $(0.09 \%$ sodium chloride solution) by oral gavage at $1 \mathrm{~h}$ after TBI, and then once per day. All procedures were approved by the Ethics Committee of Tianjin Medical University (Tianjin, China). All experiments were performed by investigators blinded to the study groups, which were only revealed at the end of the analyses.

\section{Cerebral Infarction Assay}

Brain infarction volume was measured using triphenyltetrazolium chloride (TTC) staining on brain tissues collected at $72 \mathrm{~h}$ after TBI (34). Under deep anesthesia, mice were perfused transcardially with cold PBS. The brain tissue was then obtained, incubated immediately for $15 \mathrm{~min}$ at $-20^{\circ} \mathrm{C}$. Afterwards, the frozen whole brains were sliced into 1.0-mm sections using a mouse brain slicer (Zivic Instruments, Pittsburgh, PA, USA). The brain slices were incubated in $2 \%$ (w/v) TTC (Sigma-Aldrich, St. Louis, MO, USA) dissolved in PBS for $30 \mathrm{~min}$ at $37^{\circ} \mathrm{C}$ and then transferred to $5 \%$ formaldehyde solution for fixation. The infarct area was determined by measuring the regions that lacked TTC staining, which was quantified using Image J analysis, as previously described (35).

\section{Rotarod Test}

The rotarod test was used to evaluate systemic motor function, especially coordination and balance. Mice were placed on a rotarod apparatus set at an accelerating rotational speed of $0-40$ revolutions per minute, as previously reported (36). The rod's rotational speed was accelerated from 0 to $40 \mathrm{rpm}$. The time each mouse spent on the rod was recorded. Three repeat trials were performed for each animal, and the results were calculated as the average of the three trials.

\section{Modified Neurological Severity Score}

As previously reported (36), the modified Neurological Severity Score (mNSS) was used to assess posttraumatic neurological function. The mNSS involves motor, sensory, balance beam, and reflex tests, with scores ranging from 0 (normal function) to 18 (maximal deficit). In the present study, mNSS tests were performed on days $1,3,5$, and 7 post-TBI. mNSS assessments were performed by two observers blinded to the treatment groups.

\section{Moderate CCI Model}

Controlled cortical impact (CCI) injury was performed as previously described (37). Moderate CCI injury was induced using an electromagnetically-driven CCI injury device (eCCI6.3 device; Custom Design \& Fabrication, USA) and a 2-mmdiameter round impact tip (impact depth $1.2 \mathrm{~mm}$, speed 5.0 $\mathrm{m} / \mathrm{s}$, dwell time $150 \mathrm{~ms}$ ). Mice in the sham group received all procedures apart from CCI.

\section{Western Blotting}

Western blotting (WB) was performed as previously described (38). PVDF membranes were blocked with 5\% skim milk for $2 \mathrm{~h}$ at room temperature, and then incubated with primary antibodies against caspase-3 (1:1,000; CST, USA), ZO-1 (1:1,000; Cambridge, MA, USA), $\beta$-actin (1:1,000; ZSGB-BIO, China), ATF4 (1:1,000; CST), eIF2 $\alpha$ (1:1,000; CST), or NeuN (1:1,000; Abcam, UK) at $4{ }^{\circ} \mathrm{C}$ overnight. After incubation with secondary antibodies (goat anti-mouse or anti-rabbit IgG, 1:5,000; ZSGB$\mathrm{BIO})$ at room temperature for $1 \mathrm{~h}$, the blots were then processed and analyzed.

\section{Morris Water Maze}

The Morris water maze test was used to assess the spatial learning and memory function of mice, as previously reported (39). All mice were trained for five continuous days starting at 14 days post-TBI. The number of crosses into the platform quadrant and the percentage of time spent in the platform quadrant in 60-s intervals were recorded. 
TABLE 1 | Comparison of the protective effects of sartans on TBI mice.

\begin{tabular}{|c|c|c|c|c|c|}
\hline Sartan species & Behavioral outcomes & BBB improvement & Neuroprotective effect & Involved mechanisms & Reference \\
\hline Candesartan & Ameliorated cognitive impairment & No mention & Positive & AT1R blocking and PPAR $\gamma$ activating & $(18)$ \\
\hline Telmisartan & No effect & No mention & Positive & AT1R blocking and PPAR $\gamma$ activating & $(18)$ \\
\hline Candesartan & Positive & Positive & Positive & No mention & $(25)$ \\
\hline Candesartan & Positive & No mention & Positive & No mention & $(15)$ \\
\hline Losartan & No mention & Positive & Positive & TGF- $\beta$ signaling blocking & (26) \\
\hline Candesartan & Positive & No mention & Positive & AT1R blocking and PPAR $\gamma$ activating & $(14)$ \\
\hline
\end{tabular}

A

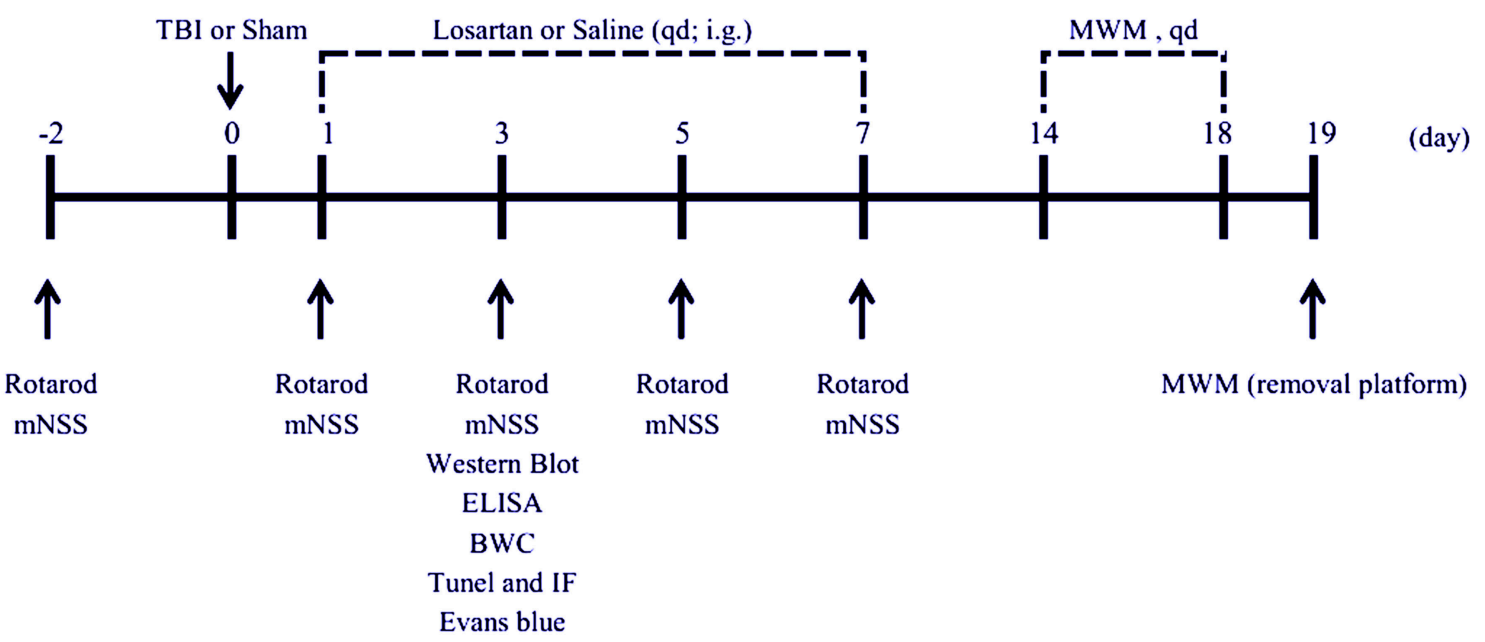

B

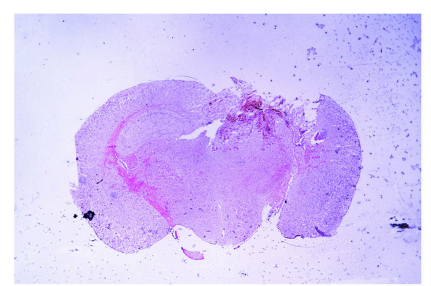

TBI+Losartan 0mg/kg
C

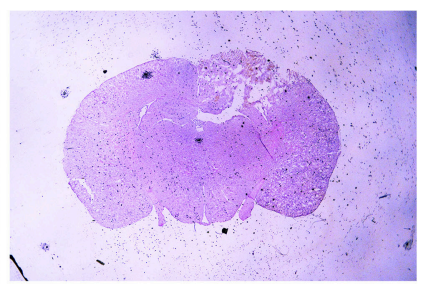

TBI+Losartan $1 \mathrm{mg} / \mathrm{kg}$
D

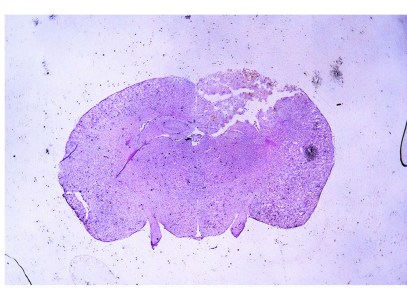

TBI+Losartan 3mg/kg
E

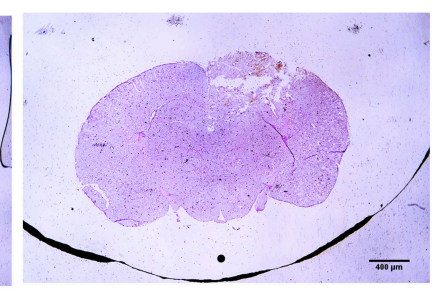

TBI+Losartan 10mg/kg

FIGURE 1 | (A) Timeline of the animal experiment. (B) The volume of lesion of TBI mice after 1 day. (C-E) After receiving different doses of losartan (1, 3, 10 mg/kg), representative HE staining of brain tissues section at 1 day after TBI.

\section{ELISA}

Total protein concentration was measured using a BCA protein assay kit (Beyotime, Shanghai, China). At 3 days after TBI, the levels of interleukin-10 (IL-10) and tumor necrosis factor (TNF$\alpha$ ) in the injured cortex were measured using specific ELISA kits (R\&D systems, USA), as per the manufacturer's instructions.

\section{TUNEL Assay}

The TUNEL staining procedure was then performed as previously described (40). TUNEL and immunofluorescence staining of neuronal nuclei (NeuN) were combined to evaluate neuronal apoptosis. Frozen sections were prepared 3 days postTBI and were incubated with an anti-NeuN antibody (1:500, Abcam, Cambridge, MA, USA) at $4^{\circ} \mathrm{C}$ overnight. After $1 \mathrm{~h}$ incubation with Alexa Fluor-conjugated anti-rabbit or antimouse IgG (1:500, Thermo Fisher Scientific, Waltham, MA, USA), $50 \mu \mathrm{l}$ of TUNEL mixture was added, and the sections were incubated for $1 \mathrm{~h}$ at $37^{\circ} \mathrm{C}$ and then incubated with DAPI. A fluorescence microscope was used to determine the number of apoptotic neuronal cells around the traumatic lesion. The neuronal apoptosis ratio was recorded for statistical analysis.

\section{EB Permeability Assay}

Evens blue (EB) dye extravasation was used to evaluate BBB permeability, as previously described (40). Briefly, mice were injected with $100 \mu \mathrm{l}$ of $4 \% \mathrm{~EB}$, and then perfused with phosphate buffered saline. The ipsilateral hemisphere was then dissected, weighed, and homogenized in $0.1 \mathrm{~g} / \mathrm{ml} \mathrm{N}, \mathrm{N}$-dimethylformamide 
A

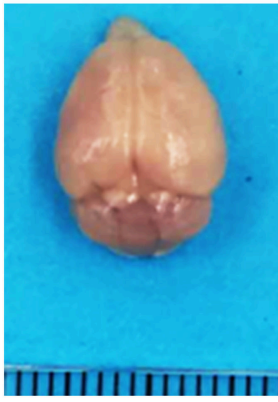

B Sham

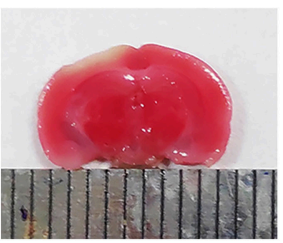

Sham

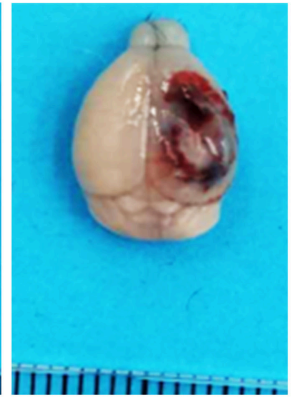

TBI+Saline

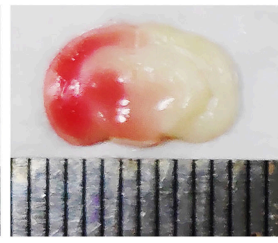

TBI+Saline

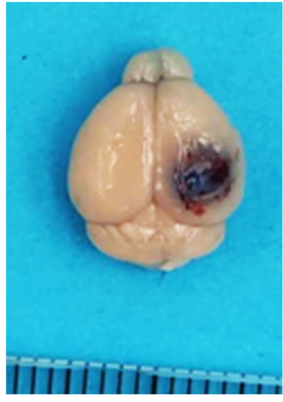

TBI+Losartan

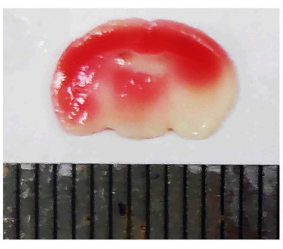

TBI+Losartan

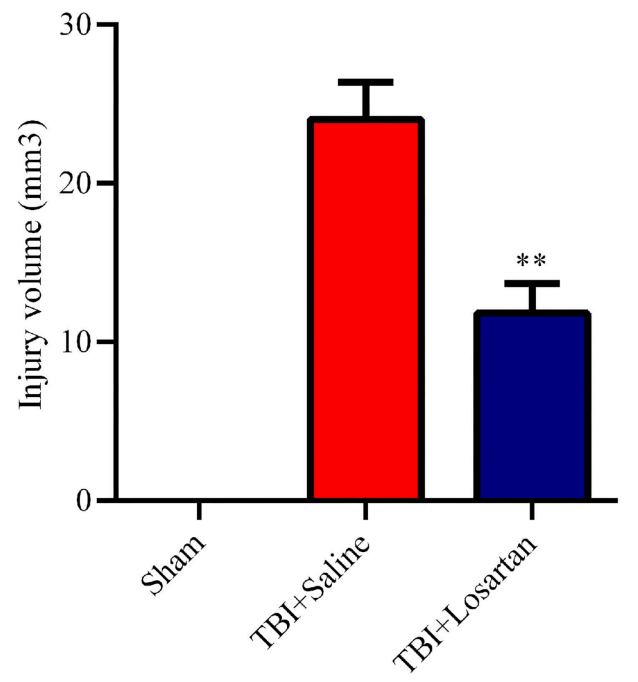

FIGURE 2 | (A) Representative images of brain tissues $1 \mathrm{~h}$ after Evans blue (EB) injection at $72 \mathrm{~h}$ after TBI. (B) Representative coronal brain sections stained with TTC and the quantification of the infarct size of mice receiving losartan or vehicle on day 3 after TBI. (C) Effect of losartan treatment on injury volume at $72 \mathrm{~h}$ after TBI. All data are expressed as the mean $\pm \mathrm{SD},{ }^{* \star} p<0.01 \mathrm{vs}$. the $\mathrm{TBI}+$ saline group.

(Sigma-Aldrich, USA), and centrifuged for $30 \mathrm{~min}$ at 3,000 revolutions per min. The supernatants were collected, and the EB content in the brain tissue was calculated from the OD values (at $610 \mathrm{~nm}$ ) using a standard curve.

\section{Measurement of Cerebral Edema}

Brain water content was measured by the wet-dry weight ratio method, as previously described (40). The percentage of brain water content was calculated as (wet weight - dry weight)/wet weight $\times 100 \%$.

\section{HE Staining}

Brain sections were fixed with paraformaldehyde and washed three times. The method of Hematoxylin-Eosin (HE) staining is carried out according to the instructions of the manufacturer (Solarbio, Beijing, China).

\section{Statistical Analysis}

A repeated-measures statistical approach was used to effectively control the false positive rate. Data are expressed as the mean \pm standard deviation. All experiments were performed in a randomized and blinded manner. SPSS statistical software (version 23.0, IBM) was used for all statistical analyses in the present study. Comparisons between two groups were analyzed by a $t$-test, while comparisons between multiple groups were analyzed by one-way ANOVA followed by the LSD and Bonferroni post-hoc test. The protein band intensity for the Western blots were determined using Image J software. Measurement of fluorescence was calculated by Photoshop. Differences were considered significant at $P<0.05$.

\section{RESULTS}

\section{Losartan Treatment Reduces Brain Injury Volume After TBI}

The timeline of the animal experiments is presented in Figure 1A. To determine the lowest and most effective dose of losartan, we treated mice $1 \mathrm{~h}$ after CCI injury and sacrificed at 1 day post-injury to assess lesion volume by $\mathrm{HE}$ staining (Figure 1B). We determined responses to doses spanning the clinical therapeutic range for losartan $(1 ; 3 ; 10 \mathrm{mg} / \mathrm{kg})$. The lowest dose of losartan (1 mg/kg; Figure $1 \mathrm{C}$ ) produced smaller reductions in lesion volume than the other doses (3 and $10 \mathrm{mg} / \mathrm{kg}$ ). Both the middle and high doses (3 and 10 $\mathrm{mg} / \mathrm{kg}$ ) of losartan were equally effective in reducing the lesion volume (Figures 1D,E). We therefore proceeded using the lowest effective dose of losartan ( $3 \mathrm{mg} / \mathrm{kg}$ ). Using EB staining, the injury volume at 3 days recovery was significant lower in the TBI + losartan group than in the TBI + saline group (Figure 2A). TTC staining confirmed that injury volume was significantly reduced in the TBI + losartan group $\left(11.80 \pm 0.94 \mathrm{~mm}^{3}\right)$ compared with the TBI + saline group $\left(24.05 \pm 1.16 \mathrm{~mm}^{3} ; P<0.01\right.$; Figures 2B,C).

\section{Losartan Treatment Attenuates Neurological and Motor Function Deficits After TBI}

The restoration of spatial memory was evaluated by the percentage of time spent in the platform quadrant and the number of crosses into the platform quadrant in 60-s intervals. 
A

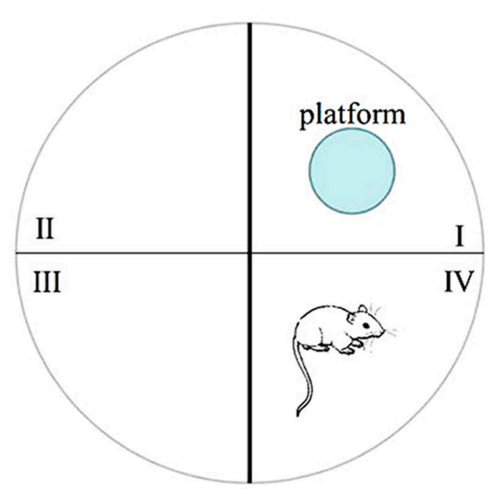

D

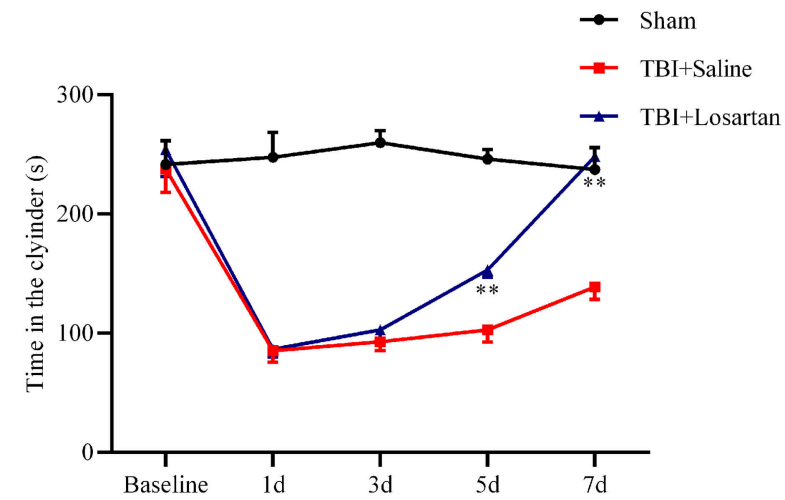

C

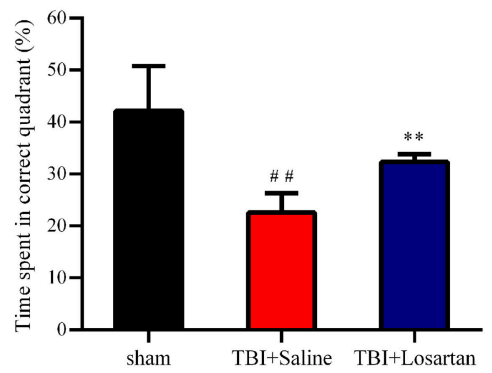

$\mathbf{E}$

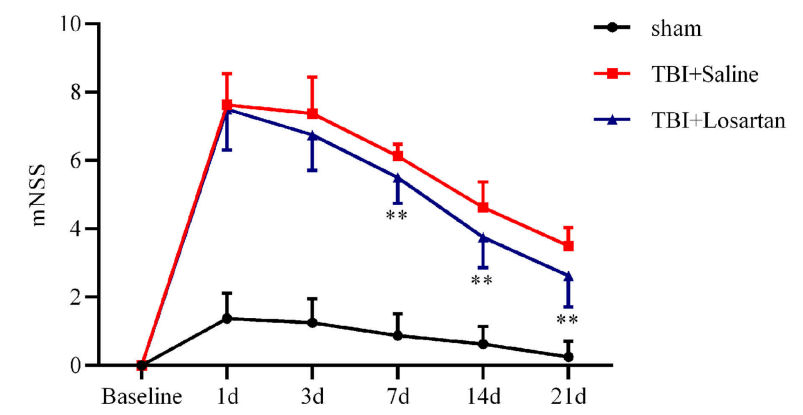

FIGURE 3 | (A) Representative images of Morris water maze in different groups, $n=8 /$ group. (B) Frequency through the platform quadrant of the mice between different groups. (C) Time percentage of the mice in the platform quadrant between different groups. (D) The duration time in the cylinder in the different groups at different time points. (E) mNSS score of mice in the different groups at different time points. All data are expressed as the mean \pm SD. $\# \# p<0.01$ vs. the sham group, ${ }^{* *} p<0.01$ vs. the TBI + Saline group.

The number of crosses into the platform quadrant was significantly lower in the TBI + saline group $(7.44 \pm 0.53)$ than in the TBI + losartan group $(10.56 \pm 0.73 ; P<0.01)$ and the sham group $(11.78 \pm 0.62 ; P<0.01$; Figures 3A,B). Similarly, the percentage of time spent in the platform quadrant was significantly lower in the TBI + saline group $(23.14 \pm 1.53 \%)$ than in the TBI + losartan group $(31.39 \pm 1.57 \% ; P<0.01)$ and the sham group ( $41.46 \pm 1.38 \% ; P<0.01$; Figures 3A,C). Overall, these data suggest that losartan treatment can improve TBI-induced cognitive deficits.

Motor coordination and balance was evaluated using the rotarod test. There were no significant difference in the time spent on the cylinder between the TBI + saline group and the TBI + losartan group at 1 day $(P=0.837)$ and 3 days $(P=0.204)$ after TBI. However, the time spent on the cylinder was significantly lower in the TBI + saline group than in the TBI + losartan group at $5 \mathrm{~d}$ and $7 \mathrm{~d}$ after TBI $(P=0.004$, and $P=0.002$, respectively). Overall, these data suggest that losartan treatment can improve the motor dysfunction caused by TBI (Figure 3D).

The neurological function of the mice was assessed by mNSS. There was a significant increase in the mNSS after TBI. At 3 days after TBI, the neurological function of mice had gradually recovered in the TBI + losartan treatment group, while the mNSS was significantly lower in the TBI + losartan treatment group than in the TBI + saline group on 3 days $(P=0.001), 5$ days $(P<0.01)$, and 7 days $(P<0.01)$ after TBI (Figure 3E).

\section{Losartan Treatment Alters Cytokine Levels in the Peri-Contusional Cortex After TBI}

ELISA was used to determine the effect of losartan on antiinflammatory (IL-10) and pro-inflammatory (TNF- $\alpha$ ) cytokine protein expression in the peri-contusional cortex. IL-10 and TNF- $\alpha$ expression were both low in the sham group. TNF- $\alpha$ expression was significantly lower in the TBI + losartan group than in the TBI + saline group $(P<0.01$; Figure 4A). Similarly, IL-10 expression was significantly higher in the TBI + losartan group than in the TBI + saline group $(P<0.01$; Figure 4B).

\section{Losartan Treatment Decreases EB Permeability and Brain Water Content and Improves BBB Integrity After TBI}

Next, we measured EB permeability and brain water content to determine the effect of losartan treatment on BBB injury 


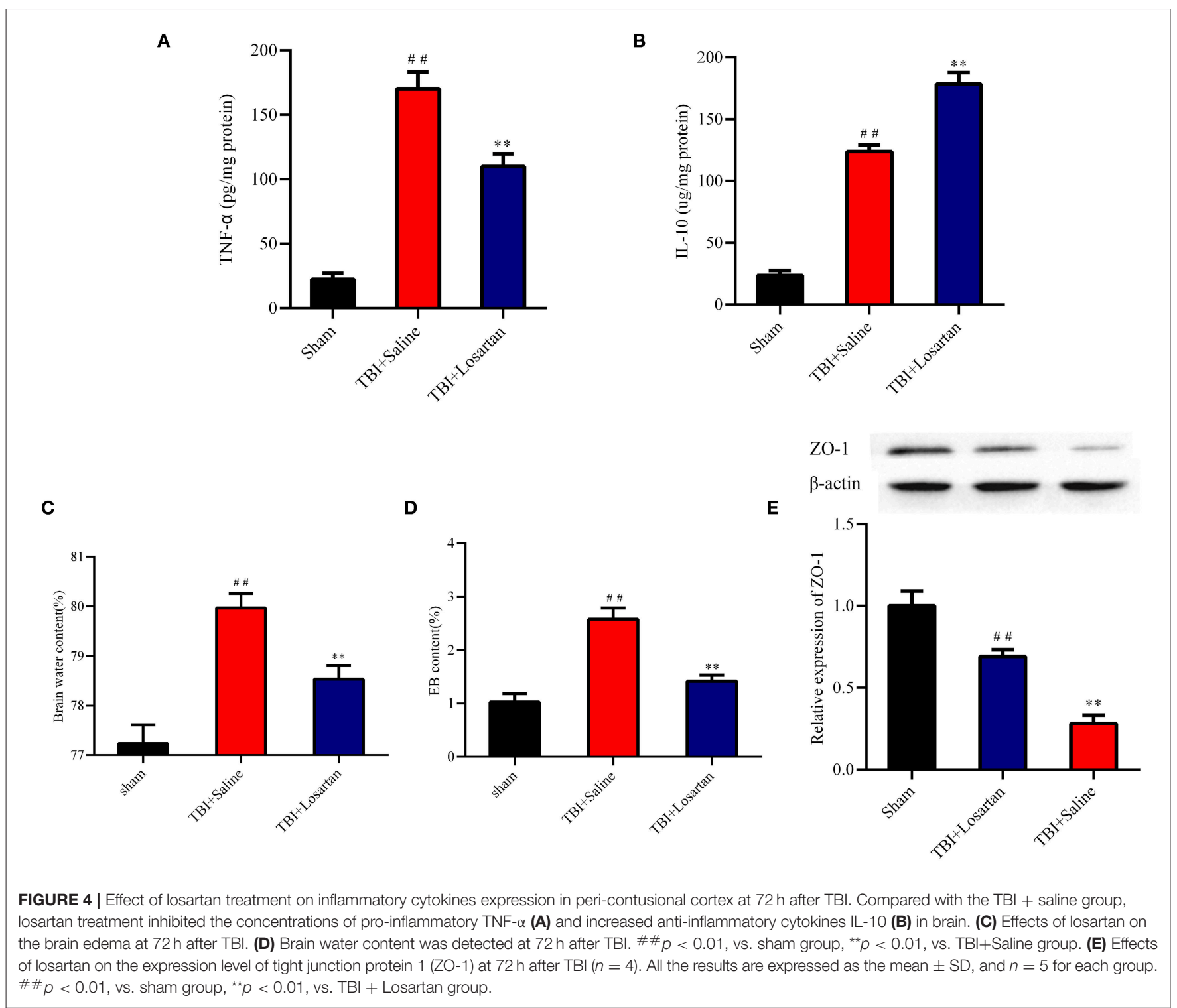

following TBI. At 3 days after TBI, there was a significant increase in brain edema in the TBI +saline group (79.97 \pm $0.12 \%)$ compared with the sham group $(77.23 \pm 0.15 \% ; P<$ 0.01 ), which was significantly reduced in the TBI + losartan group $(78.53 \% \pm 0.11 \% ; P<0.01$; Figure $4 \mathrm{C})$. Moreover, EB permeability was significantly higher in the TBI + saline group $(2.58 \pm 0.21 \mathrm{ug} / \mathrm{g})$ than in the sham group $(1.03 \pm 0.16 \mathrm{ug} / \mathrm{g}$; $P<0.01)$ and the TBI + losartan group $(1.41 \pm 0.11 \mathrm{ug} / \mathrm{g} ; P$ $<0.01$; Figure 4D). TBI is associated with an increase in BBB permeability via downregulation of the tight junction protein $\mathrm{ZO}-1$, which is an important protein that maintains the integrity of the BBB. At 3 days after TBI, there was a significant decrease in ZO-1 expression in the TBI + saline group compared with the sham group $(P<0.01)$ and the TBI + losartan group $(P<0.01$; Figure 4E).

\section{Losartan Treatment Reduces ER Stress After TBI}

TBI was associated with an increase in ER stress markers, which peaked at 3 days after TBI according to previous research (37). In our study, treatment with losartan significantly decreased the expression of ATF4 $(P<0.01$; Figure 5B) and eIF $2 \alpha(P<0.01$;

Figure 5A) compared with the TBI + saline group.

\section{Losartan Treatment Decreases Apoptosis After TBI}

Caspase-3 is a key protein in the process of cellular apoptosis. By $\mathrm{WB}$, we confirmed that the expression of caspase- 3 was significantly higher in the TBI + saline group than in the TBI + losartan group (Figure 5C). Next, the TUNEL assay and NeuN 


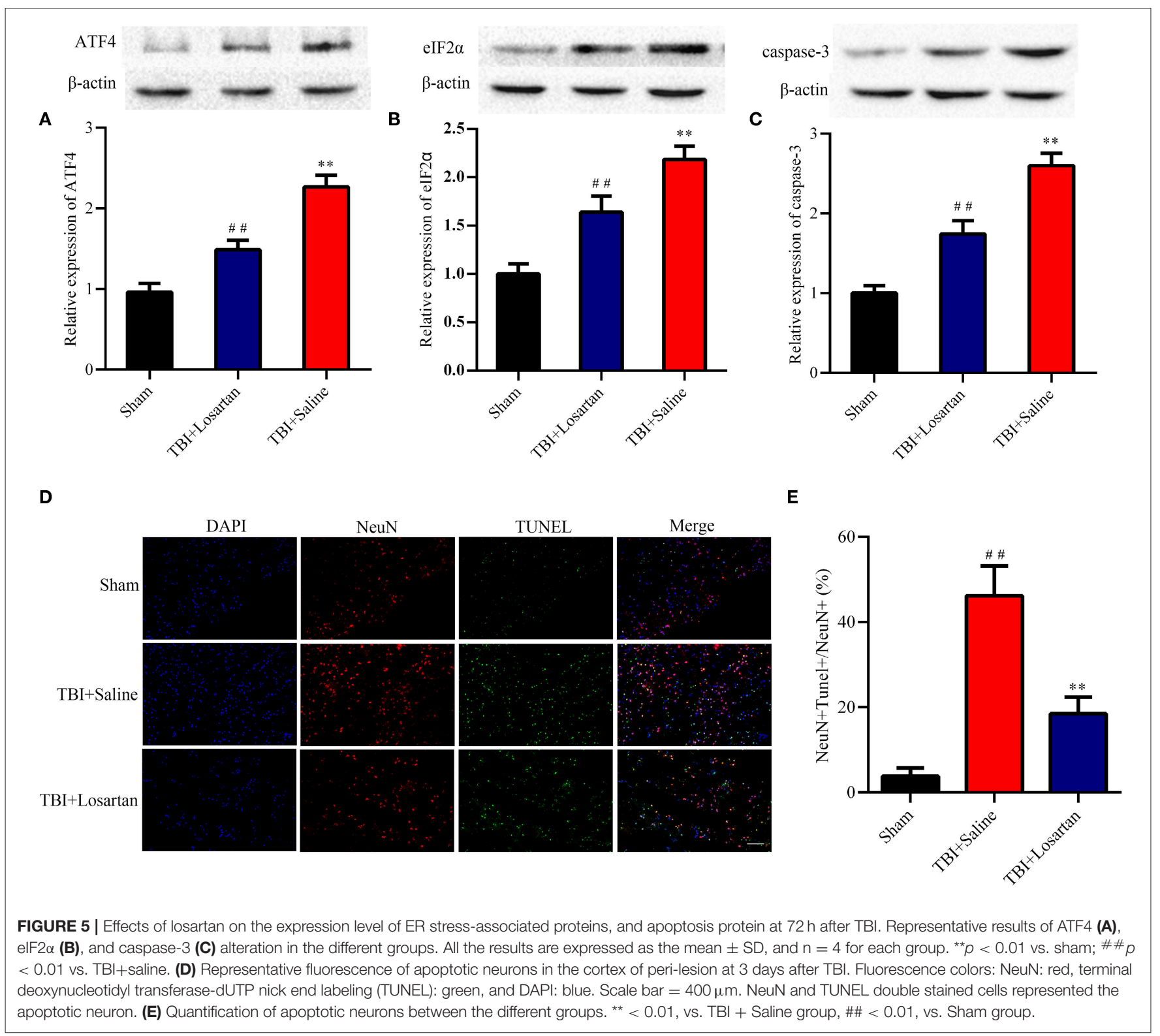

double-staining were used to quantify neuronal cell apoptosis after TBI (Figure 5D). There was a significant increase in the percentage of TUNEL-positive neurons in the lesion area of the ipsilateral cortex in the TBI + saline group compared with the sham group $(P<0.01)$ and the TBI + losartan group $(P<0.01$; Figure 5E)

\section{DISCUSSION}

AT1R are widely distributed throughout the central nervous system, and are involved with many important regulatory functions (41). It is well-established that increased AngII signaling via the AT1R has detrimental effects in stroke (42) and on cognitive function (43). In the present study, we assessed the potential of targeting AT1R signaling in ameliorating neurological damage after TBI. Abdul-Muneer et al. found that losartan can mitigate neuronal damage caused by AngII in a neuronal stretch-injury model in vitro (44). Our in vivo findings show that losartan treatment can also reduce brain lesion volume at $72 \mathrm{~h}$ after TBI, via inhibition of apoptosis in the ischemic penumbra.

The Morris water maze is a robust and reliable test of spatial learning and memory, and is strongly correlated with hippocampal synaptic plasticity and N-methyl-Daspartate (NMDA) receptor function in rodents (39). We found that losartan treatment improved both the cognitive and motor dysfunction caused by TBI, and also improved overall neurological function. It was previously reported 
that the neuroprotective action of losartan after cerebral ischemia/reperfusion injury involved inhibition of the AT1/ASK1/MKK4/JNK3 pathway in the hippocampal CA1 region (30). These findings imply that losartan has both acute and long-term benefits. However, further studies are required to examine the efficacy of long-term losartan treatment.

These is accumulating evidence that TBI is associated with neuroinflammation involving microglial and astrocyte activation, and that the neuroinflammatory cascade can control the development of cerebral edema, BBB disruption, and secondary neuronal injury (45). The result that losartan treatment significantly improve the ratio of anti-inflammatory (IL-10) to pro-inflammatory (IL-6) cytokines in the brain after TBI may be one of the mechanisms of mouse recovery. Furthermore, losartan can directly act on microglia or astrocytes, and their protective benefits were verified in comparable studies $(33,46,47)$. Previous studies have also confirmed that excessive AngII can activate rat neurons and microglia in vitro, and that losartan can block this effect $(31,44)$. Additionally, Benicky et al. reported direct anti-inflammatory effects of sartans in cultured rat microglia, cerebellar granule cells, and cerebral microvascular endothelial cells (16). Overall, these findings indicate potential mechanisms underlying the neuroprotective actions of losartan in the present study. Markers associated with glial cell activation including GFAP and Iba-1 are necessary to verify in order to obtain reliable evidence.

AngII plays a pivotal role in secondary injury after TBI. Overproduction of AngII following TBI can activate oxidative stress and caspase-3 pathways, which can result in cellular apoptosis (44). The BBB is composed of pericytes, astrocytes, endothelium, and tight junction proteins, and is surrounded by neurons $(48,49)$. After TBI, excessive oxidative stress can directly downregulate the expression of ZO-1 (50), leading to increased BBB permeability. At the same time, neuronal loss can also contribute to BBB damage. In the present study, we found that losartan treatment after TBI could block these upstream processes, and thus improve $\mathrm{BBB}$ integrity in vivo.

Previous studies have reported that candesartan and telmisartan treatment can improve recovery from TBI via a combination of PPAR $\gamma$ agonist activity and AT1R blockade (18), which may represent a mechanism of action for losartan. We also found that losartan treatment suppressed ER stress after TBI. ER stress can disturb the inflammatory response following TBI via inhibition of the nuclear factor kappa-B (NF- $\kappa \mathrm{B})$ signaling pathway (51), and lead to neuronal death through ATF4 (52). In support, the neuroprotective actions of candesartan were reported to involve inhibition of ER stress in a rotenone model of Parkinson's disease in rats (53), potentially via inhibition of the ATF4-CHOP-Puma pathway. Thus, the therapeutic effect of losartan on TBI in the present study may relate to suppression of ER stress, although further studies are required to determine the specific signaling mechanisms.

Losartan was originally used for treatment of hypotension, although it was also reported to have cardiovascular $(54,55)$ and renal protective effects (56) unrelated to blood pressure. Cerebrovascular hemodynamic changes are often observed following TBI, which are associated with poor prognosis. So, does losartan affect blood pressure? Our study found that the blood pressure of TBI mice taking losartan was a little low, but there was no statistical difference between the sham group and the TBI group (data not shown). Previous research has shown that the dose of losartan ( $3 \mathrm{mg} / \mathrm{kg}$ daily) used in the present study was low, without any significant effects on cerebral perfusion (57). Moreover, other studies confirmed that greater doses of losartan will not affect blood pressure in mice, which is consistent with our results (58). Thus, our neuroprotective actions of low-dose losartan are unlikely related to effects on blood pressure.

\section{CONCLUSION}

Our study provides new evidence that losartan exerts short- and long-term protection against secondary brain injury caused by TBI. Specifically, losartan treatment reduced neuronal apoptosis and ER stress, enhanced BBB integrity, and improved cognitive and motor function, as well as overall neurological function. These findings suggest that losartan is a potential candidate for treatment of TBI. Future studies are required to determine the specific mechanisms of action of losartan in secondary brain injury, including the signaling pathways and effects on different cell types, including astrocytes, microglia, and endothelial cells.

\section{DATA AVAILABILITY STATEMENT}

The raw data supporting the conclusions of this article will be made available by the authors, without undue reservation.

\section{ETHICS STATEMENT}

The animal study was reviewed and approved by Institutional Ethical Review Committee of The General Hospital of Tianjin Medical University.

\section{AUTHOR CONTRIBUTIONS}

JZ: conception and design of the manuscript. JX: drafting of the manuscript. YG, XL, and KL: critical revision of the manuscript for important intellectual content. XL and KL: manuscript supervision. JX, YG, QL, and ZH: final approval of the revised manuscript. All authors contributed to the article and approved the submitted version.

\section{FUNDING}

This work was supported by grants from the National Natural Science Foundation of China (grant no.81330029).

\section{ACKNOWLEDGMENTS}

We are grateful to Li Liu, Guili Yang, Weiyun Cui, and Shu Zhang from the Tianjin Neurological Institute for providing technical support. 


\section{REFERENCES}

1. Roozenbeek B, Maas AI, Menon DK. Changing patterns in the epidemiology of traumatic brain injury. Nat Rev Neurol. (2013) 9:231-6. doi: 10.1038/nrneurol.2013.22

2. Maas AI, Stocchetti N, Bullock R. Moderate and severe traumatic brain injury in adults. Lancet Neurol. (2008) 8:728-41. doi: 10.1016/S1474-4422(08)70164-9

3. Langlois JA, Sattin RW. Traumatic brain injury in the united states: Research and programs of the centers for disease control and prevention (cdc). J Head Trauma Rehabil. (2005) 20:187-8. doi: 10.1097/00001199-200505000-00001

4. Jindal A, Mahesh R, Bhatt S, Pandey D. Molecular modifications by regulating camp signaling and oxidant-antioxidant defence mechanisms, produce antidepressant-like effect: A possible mechanism of etazolate aftermaths of impact accelerated traumatic brain injury in rat model. Neurochem Int. (2017) 12:111:3-11. doi: 10.1016/j.neuint.2016.12.004

5. Wang ZG, Cheng Y, Yu XC, Ye LB, Xia QH, Johnson NR, et al. Bfgf protects against blood-brain barrier damage through junction protein regulation via pi3k-akt-racl pathway following traumatic brain injury. Mol Neurobiol. (2016) 53:7298-311. doi: 10.1007/s12035-015-9583-6

6. Yang $\mathrm{H}, \mathrm{Gu} \mathrm{ZT}$, Li L, Maegele M, Zhou BY, Li F, et al. Sirtl plays a neuroprotective role in traumatic brain injury in rats via inhibiting the p38 mapk pathway. Acta Pharmacol Sin. (2017) 38:168-81. doi: 10.1038/aps.2016.130

7. Begum G, Yan HQ, Li L, Singh A, Dixon CE, Sun D. Docosahexaenoic acid reduces er stress and abnormal protein accumulation and improves neuronal function following traumatic brain injury. J Neurosci. (2014) 34:3743-55. doi: 10.1523/JNEUROSCI.2872-13.2014

8. Shi H, Wang HL, Pu HJ, Shi YJ, Zhang J, Zhang WT, et al. Ethyl pyruvate protects against blood-brain barrier damage and improves long-term neurological outcomes in a rat model of traumatic brain injury. CNS Neurosci Ther. (2015) 21:374-84. doi: 10.1111/cns.12366

9. Hirooka Y, Kimura Y, Nozoe M, Sagara Y, Ito K, Sunagawa K. Amlodipineinduced reduction of oxidative stress in the brain is associated with sympathoinhibitory effects in stroke-prone spontaneously hypertensive rats. Hypertens Res. (2006) 29:49-56. doi: 10.1291/hypres.29.49

10. Ito $\mathrm{H}$, Takemori $\mathrm{K}$, Suzuki $\mathrm{T}$. Role of angiotensin ii type 1 receptor in the leucocytes and endothelial cells of brain microvessels in the pathogenesis of hypertensive cerebral injury. J Hypertens. (2001) 19:591-7. doi: 10.1097/00004872-200103001-00011

11. Landmesser U, Cai H, Dikalov S, McCann L, Hwang J, Jo H, et al. Role of $\mathrm{p} 47$ (phox) in vascular oxidative stress and hypertension caused by angiotensin ii. Hypertension. (2002) 40:511-5. doi: 10.1161/01.hyp.0000032100.23772.98

12. Rueckschloss U, Quinn MT, Holtz J, Morawietz H. Dose-dependent regulation of $\operatorname{nad}(\mathrm{p}) \mathrm{h}$ oxidase expression by angiotensin ii in human endothelial cells: Protective effect of angiotensin ii type 1 receptor blockade in patients with coronary artery disease. Arterioscler Thromb Vasc Biol. (2002) 22:1845-51. doi: 10.1161/01.atv.0000035392.38687.65

13. Saavedra JM, Sánchez-Lemus E, Benicky J. Blockade of brain angiotensin ii at1 receptors ameliorates stress, anxiety, brain inflammation and ischemia: Therapeutic implications. Psychoneuroendocrinology. (2011) 36:118. doi: 10.1016/j.psyneuen.2010.10.001

14. Villapol S, Yaszemski AK, Logan TT, Sánchez-Lemus E, Saavedra JM, Symes AJ. Candesartan, an angiotensin ii at1-receptor blocker and ppar- $\gamma$ agonist, reduces lesion volume and improves motor and memory function after traumatic brain injury in mice. Neuropsychopharmacology. (2012) 37:2817-29. doi: $10.1038 / \mathrm{npp} .2012 .152$

15. Timaru-Kast R, Wyschkon S, Luh C, Schaible EV, Lehmann F, Merk P, et al. Delayed inhibition of angiotensin ii receptor type 1 reduces secondary brain damage and improves functional recovery after experimental brain trauma*. Critical Care Med. (2012) 40:935-44. doi: 10.1097/CCM.0b013e3182 2f08b9

16. Benicky J, Sanchez-Lemus E, Honda M, Pang T, Orecna M, Wang J, et al. Angiotensin ii at1 receptor blockade ameliorates brain inflammation. Neuropsychopharmacology. (2011) 36:857-70. doi: 10.1038/npp.2010.225

17. Burnier M, Brunner HR. Angiotensin ii receptor antagonists. Lancet. (2000) 355:637-45. doi: 10.1016/s0140-6736(99)10365-9
18. Villapol S, Balarezo MG, Affram K, Saavedra JM, Symes AJ. Neurorestoration after traumatic brain injury through angiotensin ii receptor blockage. Brain. (2015) 138:3299-315. doi: 10.1093/brain/awv172

19. Torika N, Asraf K, Apte RN, Fleisher-Berkovich S. Candesartan ameliorates brain inflammation associated with alzheimer's disease. CNS Neurosci Therap. (2018) 24:231-42. doi: 10.1111/cns.12802

20. Li T, Zhang YR, Zhu BH, Wu CF, Chen Y. Telmisartan regulates the development of cerebral ischemia by alleviating endoplasmic reticulum stress. Die Pharmazie. (2018) 73:585-8. doi: 10.1691/ph.2018.8592

21. Abdel-Fattah MM, Messiha BAS, Mansour AM. Modulation of brain ace and ace 2 may be a promising protective strategy against cerebral ischemia/reperfusion injury: An experimental trial in rats. Naunyn-Schmiedeberg's Arch Pharmacol. (2018) 391:1003-20. doi: 10.1007/s00210-018-1523-3

22. Wang ZF, Li J, Ma C, Huang C, Li ZQ. Telmisartan ameliorates a $\beta$ oligomerinduced inflammation via ppar $/$ pten pathway in bv2 microglial cells. Biochem Pharmacol. (2020) 171:113674. doi: 10.1016/j.bcp.2019.113674

23. Elkahloun AG, Rodriguez Y, Alaiyed S, Wenzel E, Saavedra JM. Telmisartan protects a microglia cell line from lps injury beyond at 1 receptor blockade or ppar $\gamma$ activation. Mol Neurobiol. (2019) 56:3193-210. doi: 10.1007/s12035-018-1300-9

24. Bhat SA, Goel R, Shukla S, Shukla R, Hanif K. Angiotensin receptor blockade by inhibiting glial activation promotes hippocampal neurogenesis via activation of wnt/ $\beta$-catenin signaling in hypertension. Mol Neurobiol. (2018) 55:5282-98. doi: 10.1007/s12035-017-0754-5

25. Khaksari M, Rajizadeh MA, Bejeshk MA, Soltani Z, Motamedi S, Moramdi $F$, et al. Does inhibition of angiotensin function cause neuroprotection in diffuse traumatic brain injury? Iran J Basic Med Sci. (2018) 21:615-20. doi: 10.22038/IJBMS.2018.26586.6512

26. Friedman A, Bar-Klein G, Serlin Y, Parmet Y, Heinemann U, Kaufer D. Should losartan be administered following brain injury? Expert Rev Neurother. (2014) 14:1365-75. doi: 10.1586/14737175.2014.972945

27. Benigni A, Cassis P, Remuzzi G. Angiotensin ii revisited: New roles in inflammation, immunology and aging. EMBO Mol Med. (2010) 2:247-57. doi: 10.1002/emmm.201000080

28. Moulder JE, Cohen EP, Fish BL. Captopril and losartan for mitigation of renal injury caused by single-dose total-body irradiation. Radiat Res. (2011) 175:29-36. doi: 10.1667/RR2400.1

29. Sadoshima J. Novel at(1) receptor-independent functions of losartan. Circ Res. (2002) 90:754-6. doi: 10.1161/01.res.0000016843.82450.8c

30. Zhang TL, Fu JL, Geng Z, Yang JJ, Sun XJ. The neuroprotective effect of losartan through inhibiting at1/ask1/mkk4/jnk3 pathway following cerebral $\mathrm{i} / \mathrm{r}$ in rat hippocampal cal region. CNS Neurosci Ther. (2012) 18:981-7. doi: $10.1111 / \mathrm{cns} .12015$

31. Miyoshi M, Miyano K, Moriyama N, Taniguchi M, Watanabe T. Angiotensin type 1 receptor antagonist inhibits lipopolysaccharide-induced stimulation of rat microglial cells by suppressing nuclear factor kappab and activator protein-1 activation. Eur J Neurosci. (2008) 27:343-51. doi: 10.1111/j.1460-9568.2007.06014.x

32. Li Z, Bains JS, Ferguson AV. Functional evidence that the angiotensin antagonist losartan crosses the blood-brain barrier in the rat. Brain Res Bull. (1993) 30:33-9. doi: 10.1016/0361-9230(93)90036-b

33. Salmani H, Hosseini M, Beheshti F, Baghcheghi Y, Sadeghnia HR, Soukhtanloo M, et al. Angiotensin receptor blocker, losartan ameliorates neuroinflammation and behavioral consequences of lipopolysaccharide injection. Life Sci. (2018) 203:161-70. doi: 10.1016/j.lfs.2018.04.033

34. Chen W, Zheng P, Hong T, Wang Y, Liu N, He B, et al. Astrocytesderived exosomes induce neuronal recovery after traumatic brain injury via delivering gap junction alpha 1-20k. J Tissue Eng Regen Med. (2020) 14:412-23. doi: 10.1002/term.3002

35. Gan Y, Liu Q, Wu W, Yin JX, Bai XF, Shen R, et al. Ischemic neurons recruit natural killer cells that accelerate brain infarction. Proc Natl Acad Sci USA. (2014) 111:2704-9. doi: 10.1073/pnas.1315943111

36. Xu X, Gao W, Cheng S, Yin D, Li F, Wu Y, et al. Anti-inflammatory and immunomodulatory mechanisms of atorvastatin in a murine model of traumatic brain injury. J Neuroinflammation. (2017) 14:167. doi: 10.1186/s12974-017-0934-2 
37. Sun D, Gu G, Wang J, Chai Y, Fan Y, Yang M, et al. Administration of tauroursodeoxycholic acid attenuates early brain injury via akt pathway activation. Front Cell Neurosci. (2017) 11:193. doi: 10.3389/fncel.2017.00193 eCollection 2017

38. Gao W, Li F, Liu L, Xu X, Zhang B, Wu Y, et al. Endothelial colonyforming cell-derived exosomes restore blood-brain barrier continuity in mice subjected to traumatic brain injury. Exp Neurol. (2018) 307:99-108. doi: 10.1016/j.expneurol.2018.06.001

39. Vorhees CV, Williams MT. Morris water maze: Procedures for assessing spatial and related forms of learning and memory. Nat Protoc. (2006) 1:84858. doi: 10.1038/nprot.2006.116

40. Shen J, Xin W, Li Q, Gao Y, Yuan L, Zhang J. Methylene blue reduces neuronal apoptosis and improves blood-brain barrier integrity after traumatic brain injury. Front Neurol. (2019) 10:1133. doi: 10.3389/fneur.2019.01133 eCollection 2019

41. Sumners C, Horiuchi M, Widdop RE, McCarthy C, Unger T, Steckelings UM. Protective arms of the renin-angiotensin-system in neurological disease. Clin Exp Pharmacol Physiol. (2013) 40:580-8. doi: 10.1111/1440-1681.12137

42. Phillips MI, de Oliveira EM. Brain renin angiotensin in disease. J Mol Med. (2008) 86:715-22. doi: 10.1007/s00109-008-0331-5

43. Mogi M, Iwanami J, Horiuchi M. Roles of brain angiotensin ii in cognitive function and dementia. Int J Hypertens. (2012) 2012:169649. doi: $10.1155 / 2012 / 169649$

44. Abdul-Muneer PM, Bhowmick S, Briski N. Angiotensin ii causes neuronal damage in stretch-injured neurons: Protective effects of losartan, an angiotensin t1 receptor blocker. Mol Neurobiol. (2018) 55:5901-12. doi: $10.1007 / \mathrm{s} 12035-017-0812-\mathrm{Z}$

45. Gao W, Zhao Z, Yu G, Zhou Z, Zhou Y, Hu T, et al. Vegi attenuates the inflammatory injury and disruption of blood-brain barrier partly by suppressing the tlr4/nf-kappab signaling pathway in experimental traumatic brain injury. Brain Res. (2015) 1622:230-9. doi: 10.1016/j.brainres.2015. 04.035

46. Xu Y, Xu Y, Wang Y, Wang Y, He L, Jiang Z, et al. Telmisartan prevention of lps-induced microglia activation involves $\mathrm{m} 2$ microglia polarization via camkkbeta-dependent ampk activation. Brain Behav Immun. (2015) 50:298313. doi: 10.1016/j.bbi.2015.07.015

47. Hong S, JianCheng H, JiaWen W, ShuQin Z, GuiLian Z, HaiQin W, et al. Losartan inhibits development of spontaneous recurrent seizures by preventing astrocyte activation and attenuating blood-brain barrier permeability following pilocarpine-induced status epilepticus. Brain Res Bull. (2019) 149:251-9. doi: 10.1016/j.brainresbull.2019.05.002

48. Serlin Y, Shelef I, Knyazer B, Friedman A. Anatomy and physiology of the blood-brain barrier. Semin Cell Dev Biol. (2015) 38:2-6. doi: 10.1016/j.semcdb.2015.01.002

49. Sweeney MD, Zhao Z, Montagne A, Nelson AR, Zlokovic BV. Blood-brain barrier: From physiology to disease and back. Physiol Rev. (2019) 99:21-78. doi: 10.1152/physrev.00050.2017
50. Villapol S, Saavedra JM. Neuroprotective effects of angiotensin receptor blockers. Am J Hypertens. (2015) 28:289-99. doi: 10.1093/ajh/hpu197

51. Logsdon AF, Lucke-Wold BP, Nguyen L, Matsumoto RR, Turner RC, Rosen CL, et al. Salubrinal reduces oxidative stress, neuroinflammation and impulsive-like behavior in a rodent model of traumatic brain injury. Brain Res. (2016) 1643:140-51. doi: 10.1016/j.brainres.2016.04.063

52. Nakka VP, Prakash-Babu P, Vemuganti R. Crosstalk between endoplasmic reticulum stress, oxidative stress, and autophagy: Potential therapeutic targets for acute cns injuries. Mol Neurobiol. (2016) 53:532-44. doi: 10.1007/s12035-014-9029-6

53. Wu L, Tian YY, Shi JP, Xie W, Shi JQ, Lu J, et al. Inhibition of endoplasmic reticulum stress is involved in the neuroprotective effects of candesartan cilexitil in the rotenone rat model of parkinson's disease. Neurosci Lett. (2013) 548:50-5. doi: 10.1016/j.neulet.2013.06.008

54. Leversha S, Allen AM, May CN, Ramchandra R. Intrathecal administration of losartan reduces directly recorded cardiac sympathetic nerve activity in ovine heart failure. Hypertension. (2019) 74:896-902. doi: 10.1161/HYPERTENSIONAHA.119.12937

55. Teixido-Tura G, Forteza A, Rodriguez-Palomares J, Gonzalez Mirelis J, Gutierrez L, Sanchez V, et al. Losartan versus atenolol for prevention of aortic dilation in patients with marfan syndrome. J Am Coll Cardiol. (2018) 72:1613-8. doi: 10.1016/j.jacc.2018.07.052

56. Rukavina Mikusic NL, Kouyoumdzian NM, Uceda A, Del Mauro JS, Pandolfo M, Gironacci MM, et al. Losartan prevents the imbalance between renal dopaminergic and renin angiotensin systems induced by fructose overload. L-dopa/dopamine index as new potential biomarker of renal dysfunction. Metabolism. (2018) 85:271-85. doi: 10.1016/j.metabol.2018.04.010

57. Al-Mufti F, Amuluru K, Changa A, Lander M, Patel N, Wajswol E, et al. Traumatic brain injury and intracranial hemorrhage-induced cerebral vasospasm: A systematic review. Neurosurg Focus. (2017) 43:E14. doi: 10.3171/2017.8.FOCUS17431

58. Smeda JS, Watson D, Stuckless J, Negandhi A. Post-stroke losartan and captopril treatments arrest hemorrhagic expansion in shrsp without lowering blood pressure. Vasc Pharmacol. (2018) 111:26-35. doi: 10.1016/j.vph.2018.08.006

Conflict of Interest: The authors declare that the research was conducted in the absence of any commercial or financial relationships that could be construed as a potential conflict of interest.

Copyright (C) 2020 Xiong, Gao, Li, Li, Li, Shen, Han and Zhang. This is an open-access article distributed under the terms of the Creative Commons Attribution License (CC $B Y)$. The use, distribution or reproduction in other forums is permitted, provided the original author(s) and the copyright owner(s) are credited and that the original publication in this journal is cited, in accordance with accepted academic practice. No use, distribution or reproduction is permitted which does not comply with these terms. 